\title{
Gradhiva
}

GRADHIV

Revue d'anthropologie et d'histoire des arts

$21 \mid 2015$

Création plastique d'Haïti

\section{Quelques aspects de la nouvelle scène artistique d'Haïti}

Some aspects of the new artistic scene in Haiti

Carlo A. Célius

\section{OpenEdition}

Journals

Édition électronique

URL : http://journals.openedition.org/gradhiva/2950

DOI : 10.4000/gradhiva.2950

ISSN : $1760-849 X$

Éditeur

Musée du quai Branly Jacques Chirac

Édition imprimée

Date de publication : 1 février 2015

Pagination : 104-129

ISBN : 978-2-35744-075-3

ISSN : 0764-8928

Référence électronique

Carlo A. Célius, "Quelques aspects de la nouvelle scène artistique d'Haïti », Gradhiva [En ligne], 21 | 2015, mis en ligne le 01 février 2015, consulté le 20 avril 2019. URL : http://

journals.openedition.org/gradhiva/2950; DOI : 10.4000/gradhiva.2950

(c) musée du quai Branly 


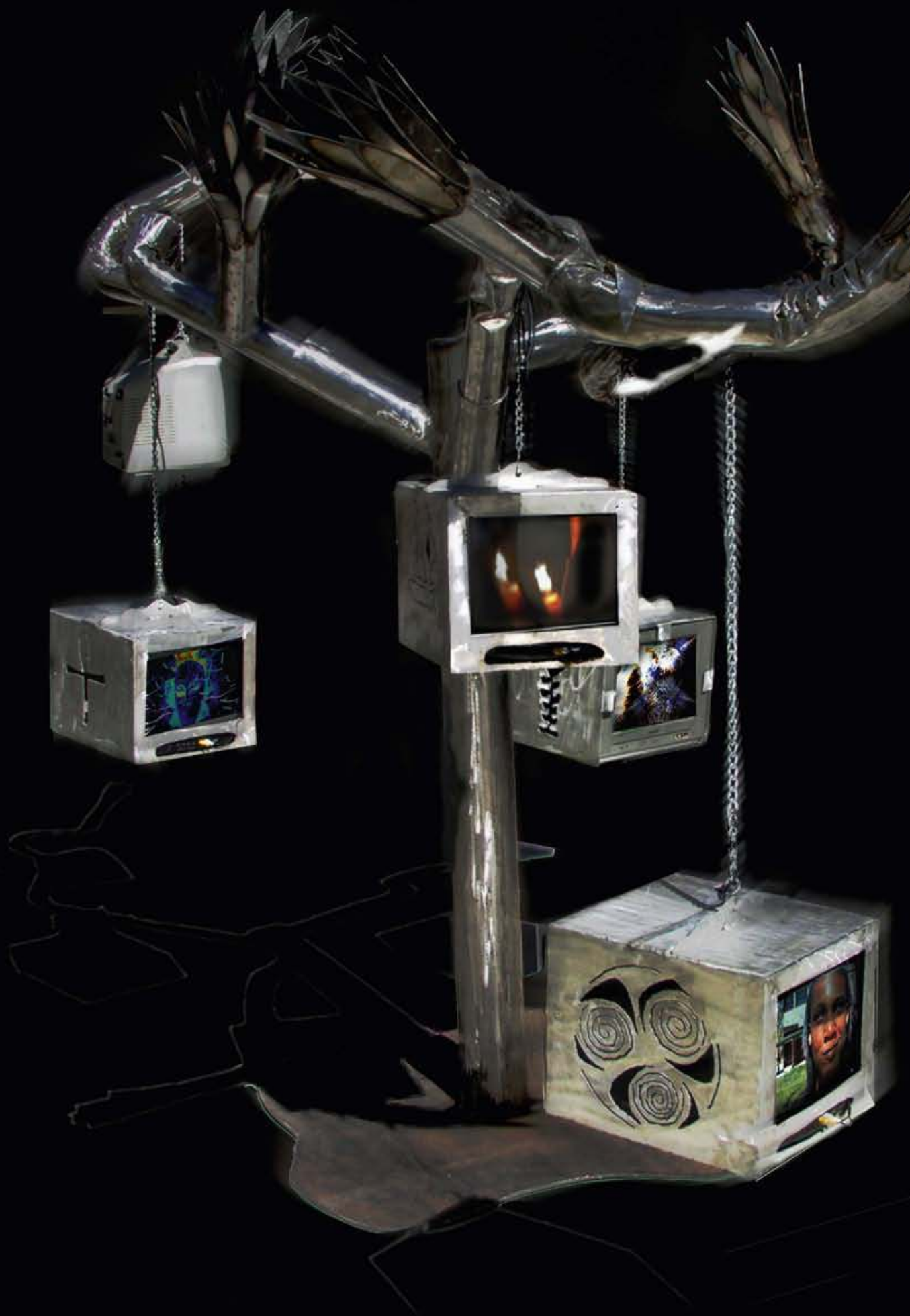
$\frac{1}{0}$
0
0
0 


\section{Quelques aspects de la nouvelle scène artistique d'Haïti}

par Carlo A. Célius

De nouveaux types d'œuvres sont apparus dans l'univers de la création plastique d'Haïti depuis les années 1980-1990. Ils participent d'une nouvelle scène dont nous allons tenter de dégager quelques caractéristiques, qui ressortiront davantage en les situant dans le processus historique global de configuration et de reconfiguration du monde artistique du pays. Ainsi apparaîtront les liens et les lignes de démarcation vis-à-vis des courants établis. En outre, l'analyse privilégiera certains aspects, comme la dynamique sociale qui sous-tend ces transformations, les références partagées et les facteurs différenciants tels que l'intérêt pour des problématiques particulières, ainsi que les usages des techniques et des matériaux. 


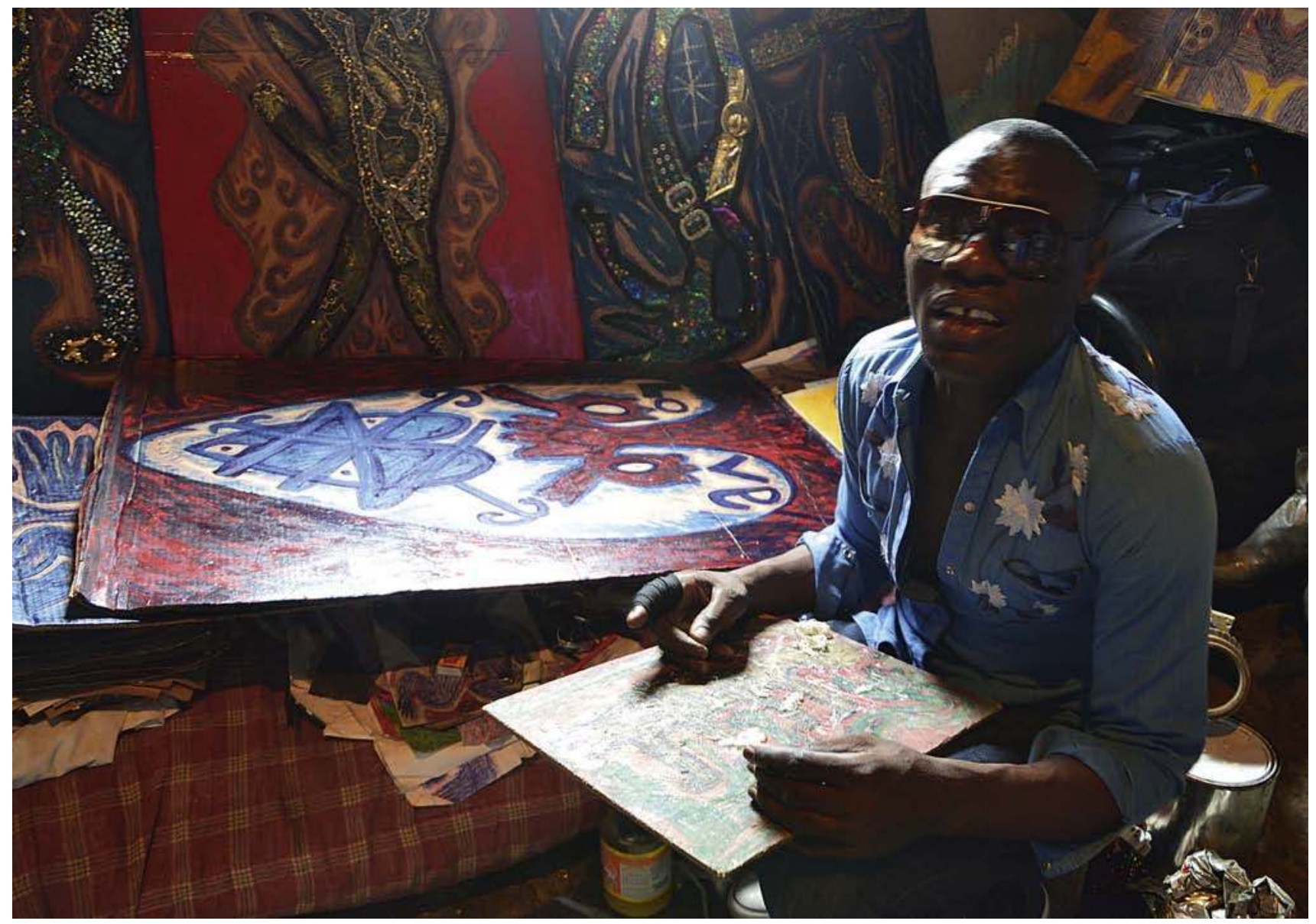

fig. 1

Guyodo dans son atelier. Au fond, des œuvres en carton, peinture et textile recyclé, mai 2014 . (c) Photo Carlo A. Célius.

\section{En ordre dispersé}

Parler de «scène artistique » permet d'évoquer plusieurs aspects du phénomène: lieu(x), espace(s), acteurs, jeux, interactions, compositions et recompositions, mais aussi a léas et improvisations. C ette métaphore est convoquée pour signifier que la mouvance artistique comporte de multiples facettes et diverses catégories de créateurs, d'intermédiaires (promoteurs, marchands, che rcheurs, crit iques) et d 'institutions dont le s int eractions, les initiatives, les formes et les niveaux d'implication sont à restituer dans la durée. À première vue, le parcours respectif des artistes, leurs démarches, leurs stratégies de promotion et l'apparition de plusieurs foyers de création indiquent que la d ynamique de la s cène artistique d'H aïti ne résulte $p$ as d'une décision unique ou d'un mot d'ordre, qu'elle ne naît pas d'un manifeste, d'une démarche ou de la demande spécifique d'une instance particulière, qu'elle ne découle pas d'une école (d'un lieu de formation), pas plus qu'elle n'en constituerait une (un regroupement autour d'une problématique, d'un projet o u d 'un s tyle). E n r éalité, pl usieurs $\mathrm{p}$ ropositions a rtistiques s ont apparues, des expérimentations isolées ont été tentées, des initiatives aux échos va riés ont é té entreprises, suivies progressivement de rencontres, d'échanges, de rapprochements, de mises en circulation sur le marché, si b ien qu'au b out du c ompte d es m anifestations c ollectives o rganisées dans le pays et à l'étranger ont fini par rendre visible et attester l'existence de la mouvance. 
S'agirait-il, avant tout, d'une affaire somme toute classique de changement de génération? On constate, en effet, que la plupart des artistes sont nés dans les années 1960, accompagnés ou rejoints par des cadets des $d$ eux $d$ écennies s uivantes. Pa rmi e ux, Ca mille $\mathrm{J}$ ean, $\mathrm{d}$ it $\mathrm{N}$ asson (1961-2008), Jean-Brunel Rocklor (1963), Mario Benjamin (1964), Élodie Barthélemy (1965), B arbara Prézeau S tephenson (1965), C éleur J ean-Hérard (1966), Patrick Ganthier, dit Killy (1966), Maksaens Denis (1968), Myrlande Constant (1968), S ergine A ndré (1969), D ubréus L hérisson (1971), F rantz Jacques, dit Guyodo (1973), Pascale Monnin (1974), Pierre-Pascal Mérisier, dit Pa skö (1974), Karim B léus (1975), D avid B oyer (1977), S ébastien J ean (1980), M anuel M athieu (1986)... Une liste exhaustive viendrait c onfirmer, quantitativement et logiquement, le critère du renouvellement générationnel. Celui-ci, to utefois, n'est p eut-être $p$ as s uffisant $c$ ar des artistes n és à I a même époque ont choisi de cheminer à travers les tendances préalablement établies et de ce fait ne s'inscrivent pas dans la mouvance quand d'autres, qui y sont affiliés ou qu'on peut y rattacher, sont nés plus tôt: c'est le cas d'André Eugène (1959), d'Édouard Duval-Carrié (1954), de Lionel St.-Eloi (1950), de Ronald Mevs (1945), de Pierrot Barra (1942-1999) ou encore de Patrick Vilaire (1941). Les parcours respectifs des artistes révèlent également d es d écalages à s ouligner. La p lupart ont d ébuté l eur $c$ arrière $p$ ar une démarche d'emblée située dans la dynamique à l'œuvre. D'autres s'y inscriront suite à une réorientation de leur projet créateur. D'autres encore s'y r etrouveront o u y s eront i ntégrés e $n$ a cquérant l e s tatut d' artiste a u travers de p ratiques a uparavant $r$ attachées à l' artisanat. Les distinctions s'observent donc à la fois suivant l'appartenance générationnelle et sociale des artistes, ainsi qu'en fonction de leur classification en tant qu'«artiste» ou «artisan » ou des jeux de passage d'une catégorie à l'autre, les connaissances et les expériences acquises dans leur parcours pouvant par ailleurs être $m$ obilisées à u $n \mathrm{n}$ iveau o $\mathrm{u}$ u $\mathrm{n}$ a utre $\mathrm{d}$ ans I eurs p ratiques a ctuelles. Quelques repères offriront une bonne esquisse des profils.

La formation initiale de Vilaire était en céramique et céramologie. Cofondateur en 1968, avec J ean-Claude G aroute (Tiga) et W ilfrid A ustin Casimir (Frido), d' un c entre de c réation et d' animation a rtistique, l' atelier Poto-Mitan, son œuvre prend une tout autre dimension à partir des années 1980 quand il s'affirme comme sculpteur, travaillant le métal. St.-Eloi, qui avait fréquenté Poto-Mitan, e st un peintre bien connu, affilié au Centre d'art de Port-au-Prince, quand, au début des années 1990, il se met à l'art volumétrique en privilégiant un matériau: I'aluminium récupéré. Mevs, qui présente $s$ es $p$ arents $c$ omme $d$ es a rtisans, s 'est I ancé $d$ ans I a p einture dans les années 1970, sur les traces du peintre Jacques Gabriel, to ut en exerçant divers métiers y c ompris a rtisanaux. Il a d éveloppé u ne œuvre comprenant peintures, sculptures, installations, performances, en manipulant de multiples matériaux (pierre, bois, papier, métal) et en expérimentant constamment différentes techniques (papier mâché, coulage, assemblage, etc.). Duval-Carrié date ses premiers essais en peinture de 1974 mais il s'est fait connaître comme peintre dans le cadre du Centre d'art au début des années 1980, avant de réorienter sa création à l a suite d'un séjour en France en 1989 o ù $\mathrm{i} I$ d écouvre e t c ommence à e xplorer l es p ossibilités o ffertes par plusieurs techniques et matériaux tels que le modelage, le moulage et la résine. Benjamin, autodidacte, est passé d'une peinture dite «hyperréaliste» 


\section{Voir Cadet 2013.}

2. La colonie française de Saint-Domingue accède à l'indépendance le $1^{\text {er }}$ janvier 1804 sous le nom d'Haïti.

3. Pour une histoire de la peinture dans le pays depuis la période coloniale jusqu'en 1980 voir Lerebours 1989 , qui est à ce jour l'étude la plus exhaustive et la mieux documentée sur le sujet. On peut également consulter Alexis 2000.
4. Tout le $x \mid x^{e}$ siècle haïtien est marqué par un discours anthropologique orienté vers la défense de l'humanité de tous les hommes, en commençant par les descendants d'esclaves. Vers la fin du siècle, on commence à s'intéresser davantage aux descriptions ethnographiques. La tendance s'amplifie et se consolide au cours de la première moitié du $x x^{\ominus}$ siècle, constituant un véritable tournant ethnologique qui procède à une redéfinition des coordonnées constitutives de la société. La collectivité est désormais pensée en termes de "communauté culturelle». Ainsi

l'indépendance et la légitimité politique, conquises de haute lutte, sur lesquelles repose le nouveau corps sociétal instauré en 1804 ne suffisent plus, comme dans la pensée duxIx ${ }^{\circledR}$ siècle, à circonscrire l'identité collective dont on perçoit désormais les composantes culturelles qu'il convient d'inventorier, d'analyser et de promouvoir. II y a la un véritable renversement paradigmatique qui a influé sur les univers de création, bouleversé les représentations collectives et les dynamiques sociopolitiques, et installé un nouveau régime de pensée.

\section{ci-contre}

fig. 2

Frantz Jacques de son nom d'artiste Guyodo,

Saint Jacques, 2009.

Verre, bois, fil métallique et textile recyclés.

Collection Jean Dornéval. Courtesy de l'artiste et des

Ateliers Jérôme. (C) Photo

Christian Nerette. représentant des figures humaines surdimensionnées à ce qu'il appelle des "portraits psychologiques ${ }^{1}$ " ainsi qu'à des installations et des performances.

Plusieurs autres artistes, qui touchent à plusieurs médiums, sont issus d'écoles d'art: Prézeau Stephenson a étudié en Haïti et au Canada, Monnin en Suisse, Barthélemy à Paris, Vladimir Cybil Charlier aux États-Unis, MarieHélène Cauvin au Canada et aux États-Unis. Eugène et Céleur ont mené leur apprentissage dans des ateliers de production d'objets d'artisanat en bois avant de pratiquer l'art de la récupération. Barra, Boyer et Lhérisson, eux aussi artistes de la récupération quoique aux orientations bien distinctes de celles des deux précédents, ont travaillé auparavant dans des ateliers de fabrication de drapo vodou. Jose Delpé et Jean Eddy Rémy ont réorienté leurs u sages $d$ u m étal d écoupé ( bidons $d$ e f uel u sagés) a près s 'y ê tre exercés selon les pratiques habituelles de leur village de Nouailles, où se concentrent des ateliers développés dans le sillage de Georges Liautaud, initiateur du courant dans les années 1950. Maksaens Denis, qui a étudié l'audiovisuel à Paris, a introduit la vidéo, tandis que s'affirment des photographes comme Henry Roy ou Josué Azor.

La multiplicité des pratiques est notable, mais l'est aussi un autre fait: la plupart des c réateurs ont étudié à l' étranger. Les mêmes, ou d'autres, y ont séjourné ou y séjournent régulièrement pour des résidences, des expositions et a utres a ctivités. Plusieurs d'entre e ux y s ont installés et y poursuivent leurs carrières. À ceux-là s'ajoutent ceux qui sont nés dans la migration ou s'y sont retrouvés très jeunes et se réclament d'Haïti: plusieurs travaillent en synergie avec des artistes qui évoluent dans le pays et participent à des manifestations collectives consacrées à la création plastique d'Haïti. Il serait difficile de les ignorer, d'autant qu'ils appartiennent à une diaspora en croissance démographique dont l'impact sur le pays est indéniable. Leurs cheminements et leurs œuvres compliquent encore la donne, à tous les points de vue. La dimension spatialement éclatée de la nouvelle scène est la touche finale mise à son caractère pluriel (parcours, expériences, modes de création, lieux de résidence...). Dans cette mouvance si diverse, quelques caractéristiques communes peuvent toutefois être dégagées, par exemple dans le partage de ressources iconographiques, l'intérêt pour des problématiques particulières ou encore les formes d'expérimentation mises en œuvre.

\section{Configurations et reconfigurations}

Évidemment, le caractère distinctif de ces caractéristiques ressortira surtout en les confrontant au passé. Mieux, c'est en inscrivant la dynamique dont e lles té moignent $d$ ans l' univers complet de la c réation p lastique $d u$ pays qu'on en aura une meilleure appréhension, qu'on percevra usages et dépassements de repères établis, reprises et réélaborations, permanences et inventions. Pendant tout le $\mathrm{XIX}^{\mathrm{e}}$ siècle $^{2}$, le s eul d omaine de la c réation plastique q ue I es é lites I égitimaient é taient I es b eaux-arts h érités $d$ e I a période colon iale ${ }^{3}$. L e r enouveau e st s urvenu e ntre l es a nnées 1930 et 1950, en lien avec le tournant ethnologique dans I'histoire intellectuelle du pays qui redéfinit la société en mettant en valeur des composantes culturelles jusque-là minorées, voire rejetées ${ }^{4}$ (Célius [dir.] 2005). Tout cela n'est pas sans rapport avec les impacts de courants de la modernité artistique 


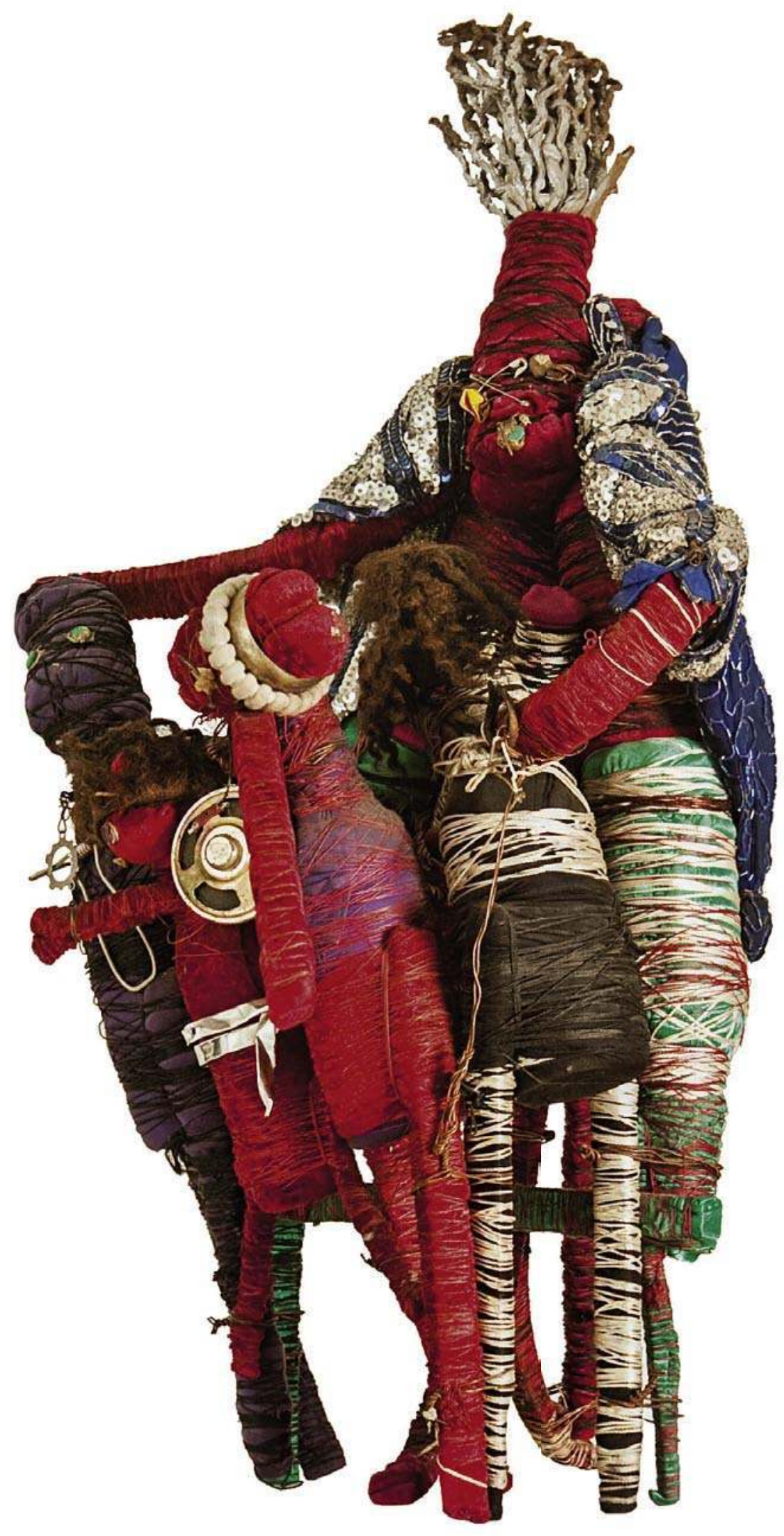


européenne où le primitivisme a joué un rôle non négligeable. On distingue au moins trois séquences significatives dans le changement survenu.

Tout d'abord, est apparu dans les années 1930 un courant pictural promouvant une modernité indigène dont l'un des gestes emblématiques a c onsisté e $\mathrm{n} I$ 'introduction $d$ ans I a t radition i nterne $d$ es b eaux-arts $d$ e thèmes $t$ irés de $c$ onditions $s$ ubalternes: $s$ ituation de $s$ pa ysans, u nivers des quartiers populaires urbains, pratiques du vodou, etc. On en mesure la p ortée quand on s ait que les b eaux-arts, imposés pendant la période coloniale comme éléments de l'appareillage symbolique de domination, ont fait l'objet d'une véritable conquête politique de la part des élites coloniales émergentes puis des élites nationales, qui s'en s ont servi c omme instrument d'affirmation de soi aux yeux de l'étranger mais aussi comme facteur de différenciation sociale sur le plan interne (Célius 2000, 2009a et 2009b). L'introduction des conditions subalternes dans l'univers d'autovalorisation des élites, même sur un mode folklorisé, a affecté et perturbé les représentations collectives dominantes. La perturbation s'est encore renforcée avec la deuxième séquence. À partir de 1945, dans des circonstances décrites ailleurs (Célius 2004 et 2007a: 311-332), on commence, au Centre d'art alors récemment créé en vue de la dynamisation des beaux-arts, à promouvoir non plus uniquement des thèmes mais des créateurs eux-mêmes issus de ces conditions sociales subalternes. Provenant de groupes sociaux infériorisés, ce qui constituait déjà une nouveauté, ces artistes n'ont de plus reçu aucune f ormation a rtistique a cadémique. I Is r éalisent a insi d es i mages acceptées en tant qu'art et pourtant complètement différentes de c e qui était jusque-là jugé tel. Les élites, hormis quelques personnalités du monde artistique et intellectuel, ont eu en premier lieu une réaction de rejet, sans pouvoir to utefois c ontrer le d éveloppement du n ouveau g enre a rtistique. Celui-ci, amorcé à la faveur de l'énonciation d'un jugement ontologique à caractère valorisant sur un tableau jusqu'alors non perçu comme une œuvre d'art (Célius 2004 et 2007a), s'instaure, stimulé par le marché externe. II se maintient, s'amplifie, se massifie même et finit par occulter, en dehors du pays, toutes les autres démarches artistiques, alors que se produit simultanément une diversification des pratiques. Des structures, a ux fortunes va riables, apparaissent, le marché interne s'organise ou se réorganise; un mode de classification et de hiérarchisation des créations se met en place, repérable notamment dans laqualification des œuvres, leur prix de vente, les stratégies adoptées pour les valoriser et les diffuser. Cette dynamique a eu pour effet, entre autres - et ceci constitue notre troisième séquence -, la reconnaissance progressive de divers domaines de création coexistant dans le pays. On a fini par établir l'existence d'un domaine de création plastique spécifique appartenant aux «arts sacrés du vodou» (Cosentino 1998 [1995]).

II faut, à ce stade, expliciter mon recours à l'expression domaine de création plastique. De nombreuses études relevant de plusieurs disciplines ont élargi les perspectives d'analyse au-delà d'une définition restrictive de l'«art». Jean-Marie Schaeffer recommande ainsi la déconstruction de la notion d'«œuvre d'art», selon lui loin d'être « une c atégorie cognitivement robuste, comme en témoignent les échecs de toute définition à ce jour». Aussi propose-t-il d'intégrer c ette notion dans une vue plus é tendue qui conçoit les œuvres d'art comme «une des cristallisations d'une classe plus 
vaste d'entités et d'événements qui renvoient à une constante fonctionnelle transculturelle et transhistorique, à savoir ce qu'en biologie et en sociologie on appelle la production des signaux à coût élevé ${ }^{5}$ ». Nul besoin de détailler tous les aspects de l'argumentation de Schaeffer pour déduire du passage cité la nécessité d'identifier, de circonscrire, de caractériser des formes de «cristallisation» pour éviter de les essentialiser, de les absolutiser. Leçon de méthode qui autorise même un retour à la notion d'œuvre d'art pour l'examiner dans ses liens avec les beaux-arts, dont l'institutionnalisation débute à la Renaissance. Rappelons que le sens spécialisé de l'expression «beauxarts » (les arts du dessin) se confirme entre les $x V I I^{e}$ et $x V I I{ }^{e}$ siècles. II se double en même temps d'un sens extensif qui fait coïncider les entités des beauxarts (écoles, musées des beaux-arts, histoire des beaux-arts) et celles de l'art (écoles et musées d'art, histoire de l'art). Une synonymie réussie d'où découle la classe essentialisée d'œuvre d'art. L'Europe étendant alors sa domination sur le monde, on comprend que les catégories des beaux-arts (élaborées par les discours qui font système avec les pratiques) aient pu servir à é valuer, cat égoriser, hié rarchiser, qual ifier/disqualifier t outes I es formes de création rencontrées ailleurs et qui s'en distinguent. S'est établie une hégémonie des beaux-arts dont il faut analyser toutes les implications, déconstruire l es " ontologies f riables " (C ometti 20 12) q ui s 'y r attachent, contrôler la projection de s c atégories a ussi b ien s ur les c réations de la période médiévale européenne (Belting 1998 [1990]) que sur celles des Taïno qui peuplaient les Grandes Antilles avant I'arrivée de Christophe Colomb. Les travaux sur les cultures visuelles ont ouvert des voies en ce sens. Ils font apparaître les bea ux-arts comme un d ispositif de production d'images parmi d'autres. Comme il demeure nécessaire de caractériser les dispositifs existants pour mieux les appréhender, je regroupe certaines formes de « cristallisation »sous l'appellation domaines de création plastique.

La création plastique comprendrait une classe d'objets résultant de la matérialisation de l'imaginaire à partir de quelques éléments de base, dont le trait (le dessin), la forme, la couleur et le volume. Le traitement technique de ces éléments, leur combinaison, les matériaux utilisés, les types d'objets qui e $n$ résultent, l eurs fo nctions, l eur m ode de va lorisation va rient d' une société à u ne autre, d'une époque à l' autre et, possiblement, d'une entité sociale à l'autre dans une même société pour une période déterminée. Ce sont ces formes d'organisation des pratiques de création plastique que j'appelle domaines de création. C'est à ce niveau que se constituent des normes, des conventions, des genres; c'est là que s'établissent la nature et les critères de jugement, que se définissent les types de discours, les fonctions des objets et leur mode de circulation. Les relations entre les domaines varient selon les périodes en raison de circonstances particulières.

Ces considé rations aident à clarifier bien des c hoses pour c e qui concerne Haïti : le contexte d'implantation du domaine des beaux-arts, les implications de leur a ppropriation et du maintien de leur hégémonie, par conséquent les effets de la remise en cause de celle-ci. On voit d'emblée qu'on ne saurait faire l'économie des relations entre le pays et l'extérieur, paramètre à $n$ e jamais o mettre $d$ ans l'analyse d es s ociétés c aribéennes, issues $d u$ pr ocès $d$ e $r$ econfiguration $d u$ m onde a morcé à $p$ artir $d$ e la fin $d u x v^{e}$ siècle. II $n$ 'est donc guère étonnant que la modernité artistique
5. "Par signal à coût élevé, on entend tout signal qui prend le contre-pied du principe d'économie régulant en général la signalisation humaine et animale. Un signal coûteux est par ailleurs nécessairement un signal honnête, au sens où il signale la qualité même qui constitue sa condition d'existence [...]. Cela explique pourquoi c'est, pour l'essentiel, dans des situations d'information incertaine que la signalisation coûteuse est utilisée [...]. » "Les signaux coûteux d'ordre artistique se rencontrent surtout dans des situations où les humains, que ce soit comme individu ou comme groupe, entrent en rapport avec une altérité, par exemple avec des esprits ou avec les ancêtres ou les morts, et, plus fondamentalement encore, lorsqu'ils se trouvent face à l'énigme de leur propre identité existentielle à l'intérieur du monde social, naturel et cosmique, etc. Bref, chaque fois que nous sommes pris dans un réseau interhumain et cosmique qui ne va pas de soi et qui nous amène à nous interroger sur nousmêmes. "Schaeffer 2008 : 174 et 175 ; voir aussi Schaeffer 2009. 


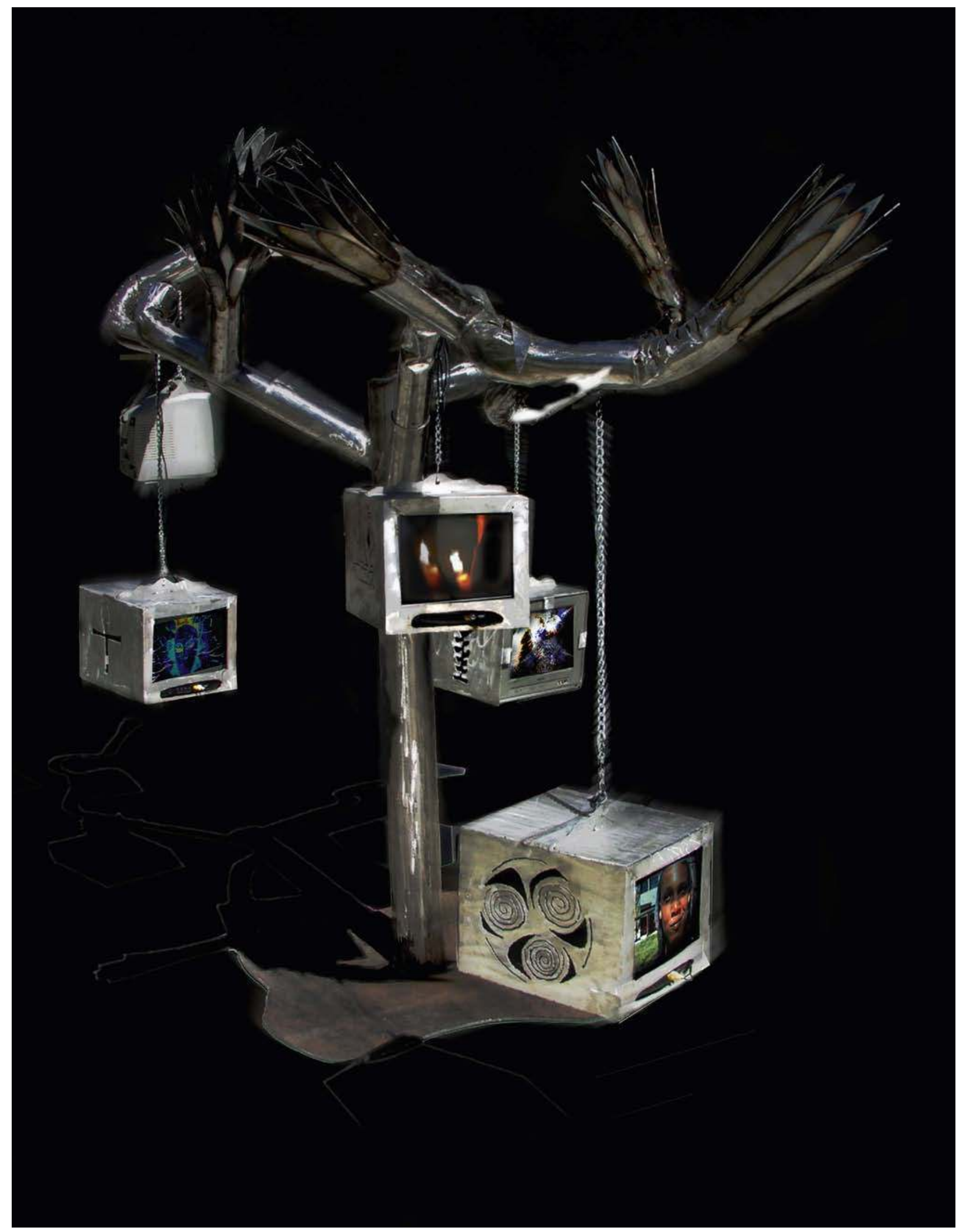


européenne et le primitivisme aient joué un rôle déterminant dans les changements survenus à partir des années 1930. Leur résonance a été facilitée par le tournant ethnologique.

Au cœur de ce renouveau, de cette redéfinition de la communauté nationale, se trouve le vodou, longtemps méprisé, refoulé, jugulé et même combattu. L'haïtianité artistique est alors mesurée par rapport à ce pôle identificatoire; l'«art haïtien» en vient à n'être qu'un «art vodou» (art religieux, sacré ou magique, selon les auteurs). D'où la confusion entre domaine de création propre au vodou et création qui s'en inspire. De fait, la thématique vodou est devenue très importante. Des symboles sont aussi enrôlés: des artistes ont cherché à s tructurer leurs œuvres en se fondant par exemple sur les dessins rituels, les vèvè. Mais la confusion des domaines maintenue dans les discours (aussi bien des promoteurs et des marchands que des artistes) aboutit, aujourd'hui encore, à la création d'œuvres aux statuts indéterminés ou conçus comme ambigus. Tout cela participe des changements survenus dans les relations entre les domaines.

À ce niveau, la nouvelle scène prolonge les séquences antérieures. Si elle est portée par des artistes nés, en majorité, entre les années 1960 et 1980 , si elle est spatialement éclatée, elle renforce, sur le plan interne, la visibilité des différents domaines de création, mais aussi leurs interactions et leurs transformations. Elle atteste, enfin, de la place significative, sinon centrale, que continue à occuper le subalterne dans la dynamique créative du pays. Au cœur de la mouvance, on retrouve, en effet, un nombre significatif de créateurs appartenant à des catégories sociales modestes, des foyers de création surgis dans des localités périphériques et des quartiers défavorisés de la région métropolitaine, comme la Grand Rue (le groupe Atis Rezistans, dont les principaux représentants sont Céleur, Eugène et Guyodo ${ }^{6}$ ), le Bel Air (avec Barra, Lhérisson et Boyer), Carrefour Feuilles (St.-Eloi, Constant), Rivière Froide (Nasson, Rocklor, Jean-Horace Salomon, dit Ti Pèlin, Bléus), ou encore Nouailles (à Croix-des-Bouquets, avec Rémy et Delpé).

\section{Ressources partagées}

Une dynamique sociale marque donc l'univers de la création plastique d'Haïti depuis la première moitié du $x x^{e}$ siècle. Elle est à la fois une constante et un ressort de renouvellement. II en est de même pour certaines sources et ressources auxquelles se réfèrent les créateurs. Qu'il s'agisse de l'oraliture ${ }^{7}$, des régimes d e c royance jusqu'alors m éprisée, infériorisée, d'événements marquants du passé, de figures héroïques, tout cela demeure des références pour des artistes de la nouvelle scène qui, souvent, célèbrent leurs prédécesseurs en en dressant des portraits, en reprenant, en citant, en réinterprétant leurs œuvres. Il y a là un univers reconnu, partagé, assumé, travaillé et retravaillé. $D$ ans I a m igration, il est m obilisé a vec d 'autres ressources pour aborder des questions de toutes sortes, qui ne se limitent pas à Haïti.

Jean Ulrick Désert (établi à Berlin après avoir migré d'Haïti aux ÉtatsUnis et vécu un moment à Paris) articule sa propre histoire de migrant avec la situation politique de son pays d'origine. II thématise également la «question raciale », notamment à travers son projet sur l'« invisibilité ostentatoire». II le fait non sans lien avec des préoccupations relevant de son orientation
6. Guyodo, qui faisait partie du collectif au début, s'en est cependant distancié.

7. L'oraliture englobe des formes discursives comme les légendes, les mythes, les contes, les proverbes, les devinettes, les formules magico-religieuses, les prières, les différents types de chansons, les sentences, les audiences, etc. Le concept, qui a connu une grande fortune dans l'univers littéraire francophone, a été proposé par Ernst Mirville, alias Pierre Bambou, en 1974 (Bambou 1974). II y revient quelques années plus tard, dans un entretien avec Pierre-Raymond Dumas, pour en préciser le contenu (Dumas 1984). Voir aussi Laroche 1991.

\section{ci-contre}

fig. 3

Maksaens Denis,

My Joshua Tree, 2008.

Installation vidéo.

(C) Maksaens Denis. 
8. Voir le site de l'artiste : http://www. jeanulrickdesert.com.

\section{ci-contre}

fig. 4

Élodie Barthélemy, CapoisLa-Mort, 2004, racine de pin, selle, étriers. Avec l'aimable autorisation de l'artiste. () Photo Manuel Choquet. sexuelle. II peut aussi convoquer l'histoire d'Haïti pour aborder des sujets se rappor tant à I 'esclavage, aux sit uations c oloniales et né ocoloniales, à l'émancipation, en intégrant des figures du "monde noir " ayant occupé des s phères allant de la politique aux arts (introduction, fig. 1). Dans son œuvre, i I s 'interroge s ur l es $m$ odalités $d$ e $c$ irculation, $d$ e r encontres e t d'échanges, les relations de genre, le racisme, les violences, les formes de domination, la situation des minorités, la reconnaissance, les transformations socioculturelles, etc., en écho (ou en concordance?) avec des formes de lutte et des courants de pensée bien reconnaissables. Ce que confirme le choix de ses œuvres pour illustrer des couvertures d'ouvrages de Stuart Hall, figure centrale des cultural studies ${ }^{8}$ (Hall 2007 et 2013).

Duval-Carrié (établi à $\mathrm{M}$ iami) ex plore é galement I a migration, c elle des Iwa (esprits vodou) par exemple. Ceux-ci voyagent, évidemment, avec leurs serviteurs. C'est donc aussi et surtout la circulation des humains qui est abordée, à commencer par celle de l'artiste lui-même qui se représente en arbre déraciné. L'interrogation $\mathrm{p}$ orte f ondamentalement su $\mathrm{r}$ le m ode d'être et le devenir dans le déplacement. Elle concerne également les images, les signes, les symboles. II y va d e leur temporalité, leur degré de stabilisation, leurs modes de transformation, leur transfiguration mais aussi, en fin de compte, des possibilités d'en inventer d'autres. Cependant, l'exemple de cette installation de Duval-Carrié (Migration of the Spirit, Figge Museum, 2006) montre surtout, et il n'est pas le seul, que l'imaginaire vodou reste une ressource très exploitée, y compris à travers des figurations et souvent des modes de présentation bien éloignés de ceux auxquels on était habitués. En té moignent é galement d es œ uvres a ussi différentes que Dessounen, sculpture en métal de Vilaire (introduction, fig. 6), Kwa Bawon, vidéo-installation de Denis ou encore Le Cercle de Freda, performance de Prézeau Stephenson.

Si c es $c$ réations $s$ e d émarquent $d$ e q uelques $r$ éférents ic onographiques $\mathrm{h}$ abituels, $\mathrm{c}$ omme $\mathrm{b}$ ien d' autres d' ailleurs, o $\mathrm{n} d$ oit $\mathrm{t}$ outefois souligner que le recours à l'imagerie chrétienne y demeure prégnant, ce qui mérite quelques explications. Dans la colonie de Saint-Domingue, la figure de l'autorité politique est légitimée par le pouvoir religieux qu'elle institue comme repère pour l'organisation de tout un pan de la vie sociale, tout cela étant lié à un mode d'organisation spatiale et de structuration temporelle. Ainsi, le catholicisme a été implanté, et est maintenu après l'indépendance, comme un cadre symbolique de référence à fort impact structurant (Célius 2014). C'est en tant qu'élément fonctionnel de ce cadre symbolique que l'imagerie religieuse chrétienne occupe une place centrale dans la culture visuelle du pays. Constituée d'un ensemble de représentations diffusées sur des supports variés, elle a c irculé et circule dans to utes les couches sociales. Comparée aux autres types d'images disponibles dans la société, elle s emble être la s eule à a voir b énéficié s ur l a d urée d' une c irculation transversale. Cela ne signifie pas qu'il y a e $u$, de to ut temps, une même intensité d e c irculation, que to ut l e m onde a to ujours é té to uché, e t a u même degré, que les modes d'appropriation ont été uniformes, etc. Il y a eu, il y a e ncore des usages différenciés, dus à plusieurs facteurs, aussi bien sociaux que r eligieux, c e qui d' ailleurs a e ntretenu u ne I ongue s ituation conflictuelle. «La profanation des choses saintes» et le sacrilège que constitue leur «mélange» avec d'autres choses sont légalement répréhensibles 


$$
1
$$


fig. 5

Jean Hérard Céleur,

Triptyque sculptural, 2006, métal, techniques mixtes. Fowler Museum à UCLA, don de Marilyn Houlberg. Photo Jessy Bernier, Perspective / Avec l'aimable autorisation des Musées de la Civilisation, Québec.

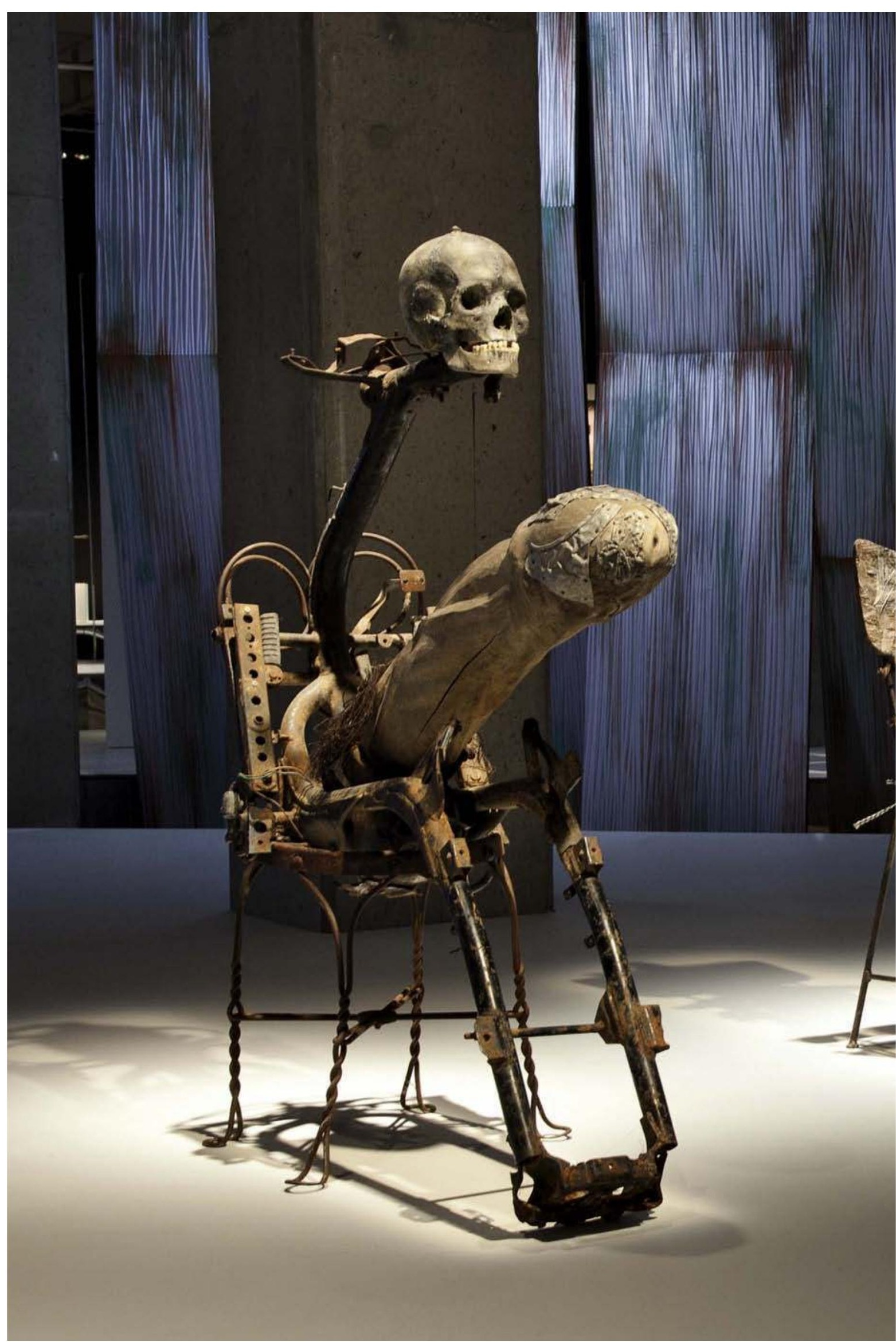




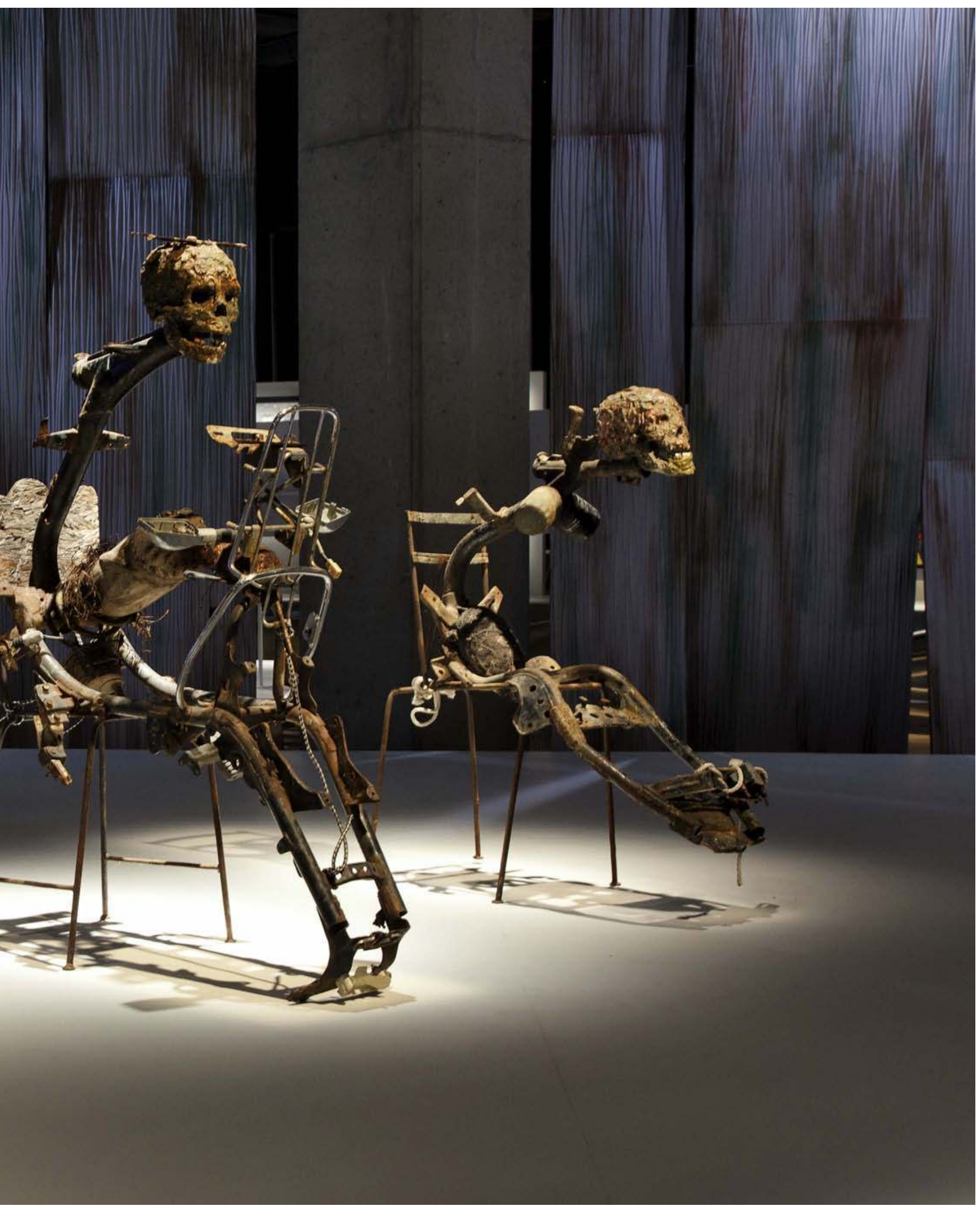


9. Arrêt du Conseil du Cap, touchant l'Empoisonneur Macandal et ses Complices, et qui ordonne la publication de l'Édit du mois de Juillet 1682 sur les poisons, du 20 janvier 1758; Arrêt de Règlement du Conseil du Cap, qui défend aux Nègres de garder des paquets appelés Macandal, ni de composer et de vendre des drogues, du 11 mars 1758. (Moreau de Saint-Méry 1784-1790: 217-218 et 222-223)

\section{ci-contre}

fig. 6

Lionel St-Eloi, L'Ange de Justice s.d., techniques mixtes. (C) Photo Roberto

Stephenson. Courtesy

Galerie Bourbon-Lally. à Saint-Domingue. Le Conseil du Cap le fait valoir à l'occasion de la retentissante affaire d'empoisonnement de 1758 d ans I aquelle est i mpliqué, à titre de leader, l'esclave marron Macandal (ou Makandal). La justice identifie l'objet scandaleux et surtout terrifiant: des «paquets ficelés » enduits d'eau et d'encens bénits, composés, entre autres éléments, de crucifix ${ }^{9} .$. Les «mélanges" perdureront et seront constamment (pendant tout le xix ${ }^{e}$ siècle et les premières années $d u x^{e}$ siècle) a $u$ cœur de dispositions juridiques et d'actions menées contre des pratiques qualifiées de superstitieuses, comme lors de la campagne dite antisuperstitieuse de 1939-1942 visant le vodou (Ramsay 2011; Clorméus 2012). II est alors remarquable de constater que c ette i magerie a j oué u n rôle dé terminant da ns I'amplification et l a transformation du domaine des beaux-arts à partir des années 1930 et 1950 en servant de référent iconographique et formel à b on nombre d'artistes, y compris ceux-là mêmes dont on a soutenu qu'ils n'en avaient pas, pour justifier I a t hèse d 'un a rt " pur », " innocent ", " premier », " pur d e t out alliage» (disait André Breton à propos d'Hector Hyppolite). Le corpus est considérable qui montre bien que bon nombre d'artistes, de toutes tendances, s'y sont référés, souvent de manière systématique et créative (Célius 2007b). II a pparaît m ême que les p ossibilités d' interprétation d es i mages disponibles et les mécanismes qui les sous-tendent constituent un facteur très important de la créativité dans le pays, surtout dans les espaces de culture subalterne. Et l'importance prise par l'imagerie chrétienne réinterprétée (qui a envahi les espaces urbains: murs, enseignes de boutiques, décors des véhicules de transport en commun appelés tap-tap, etc.) témoignerait d'un retournement de situation, si c e n'est d'une résolution, m ême p rovisoire, dans ce long conflit iconique engagé depuis Saint-Domingue.

Bon nombre d'artistes de la nouvelle scène continuent de puiser à cette source. Comme leurs prédécesseurs, ils s'en servent pour traiter des thèmes v odou, $\mathrm{c}$ hrétiens o $\mathrm{u}$ a utres. C ela p eut a ller d e l a c itation e ntière d'une chromolithographie de saint à la mise en espace d'un élément symbolique évocateur. Les démarches adoptées et les résultats obtenus varient considérablement, de la réalisation d'objets aux statuts ambigus (objets de culte ou non?) à la dérision pure. Monnin crée ainsi L'Ange de la résurrection (2006-2009) comme un «phénix qui se relève de ses cendres». L'œuvre montre un visage d'enfant «traversé par une faille piquée de diamants". "Ce visage, explique I'artiste dans une not ice d'accompagnement, nous e nseigne la nécessité de vivre avec nos cicatrices, pour faire de nos points de suture des $\mathrm{d}$ iamants é tincelants e $\mathrm{t} t$ ransformer $\mathrm{n}$ os $\mathrm{b}$ lessures e $\mathrm{n}$ a rme $\mathrm{d} e$ construction massive. » Charlier joue sur un autre registre avec Ogoun $X$, soit Malcom $X$ en /wa Ogoun, figuré d'après l'image du saint correspondant: Jacques chevauchant un cheval, brandissant son épée d'une main et tenant un drapeau de l'autre. Dans la même veine, elle a conçu Marassa Andy and Basquiat (Charlier 2005; introduction, fig. 5). Les marasa sont des jumeaux en créole haïtien. L'artiste traduit donc le compagnonnage des deux artistes, Andy Warhol et Jean-Michel Basquiat, dans le langage haïtien de la gémellité à partir d'une chromolithographie des saints Côme et Damien, correspondants catholiques des Iwa marasa. Parmi plusieurs réalisations de Cauvin retravaillant ces références, signalons La Fléchée (2007; introduction, fig. 2). Cette œuvre, qui s'inscrit dans une série sur les gangs, traite de la jeune femme pute et martyre, objet d'exploitation dans les ghettos, en convoquant 


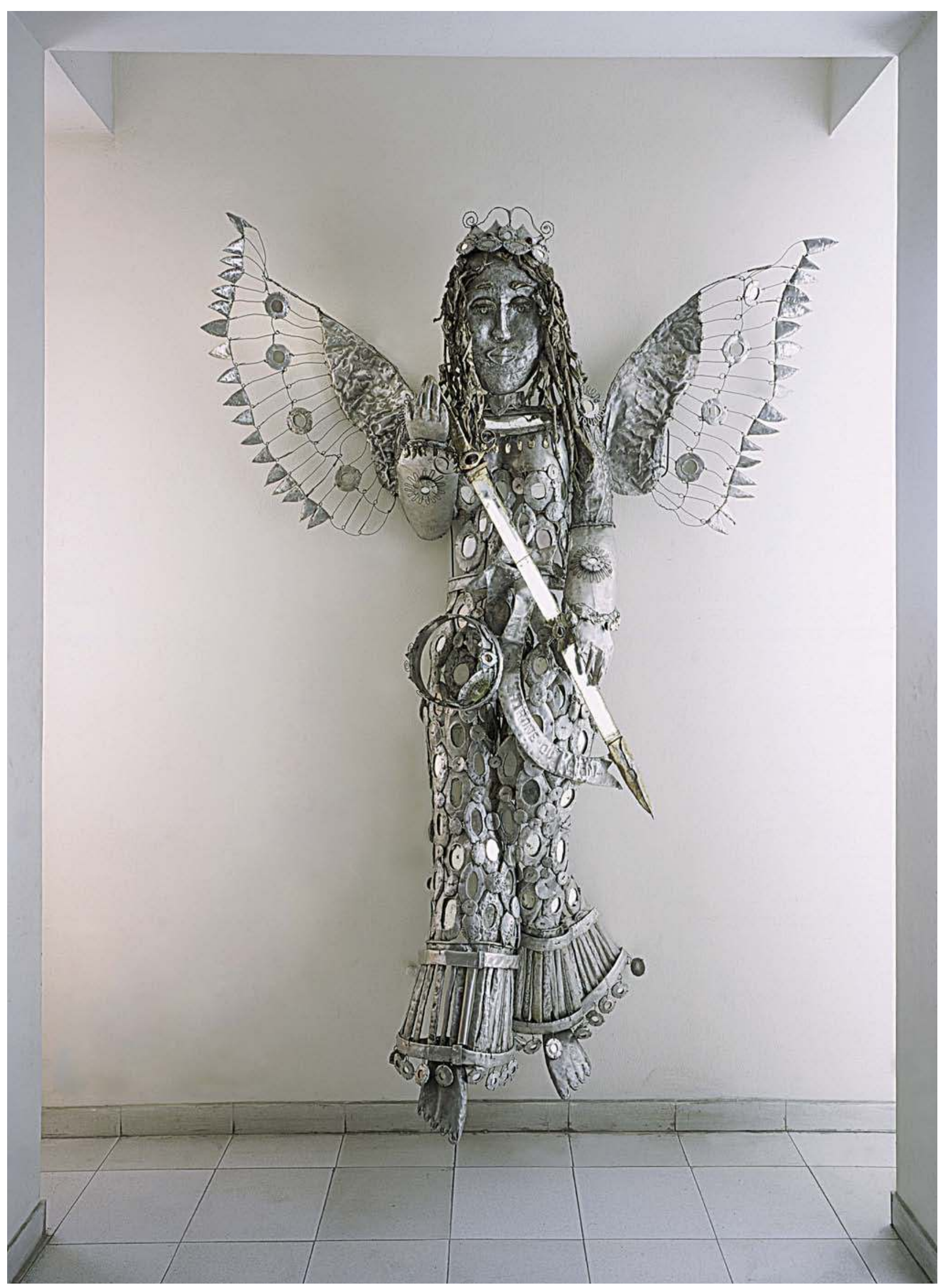


10. Élu le 16 décembre 1990, il est renversé le 30 septembre 1991 par le général Raoul Cédras. Il est rétabli dans ses fonctions le 15 octobre 1994, avec l'aide de l'armée étatsunienne. l'image de saint Sébastien. Dernier exemple, celui des artistes du collectif Atis Rezistans (de la Grand Rue) qui font un usage parfois si subtil de l'imagerie chrétienne qu'il est possible de ne pas immédiatement s'en apercevoir - telle la simple présence, persistante d'ailleurs dans leurs créations, d'une auréole.

\section{Le corps à l'œuvre}

La t ransformation e $\mathrm{t} l \mathrm{a} t$ ransfiguration $d$ e $r$ essources $p$ artagées s'opèrent en lien avec des préoccupations inédites ou plus insistantes. Ainsi il apparaît que, par rapport aux pratiques antérieures, une place plus significative est accordée au corps. Cela s'observe à trois niveaux. En premier lieu, celui de l'image du corps, du corps en image. On tend davantage à structurer les œuvres autour de la représentation du corps. Celui-ci devient objet p réoccupant d e re présentation en plus d 'être désormais I ui-même matériau $d$ ans l' acte $d$ e $c$ réation à $t$ ravers I es $p$ erformances. $D$ ans $c$ es derniers cas, le corps du regardeur-spectateur est différemment sollicité, impliqué, de même que dans les installations (fig. 3). Tout c ela $n$ 'est $p$ as sans rapport a vec un deuxième niveau, c elui du corps de l'image ou du corps image. La structuration de l'image autour de la représentation ou de la présentation du corps suscite des interventions complémentaires plus soutenues se traduisant dans un travail parfois très élaboré sur la surface, un investissement sur les factures, une recherche d'aspects, d'effets particuliers. Les deux premiers niveaux impliquent un troisième en ce qu'ils supposent des modalités de mise au travail du corps. Le corps au travail, ce sont par exemple les moyens déployés pour la réalisation et l'exposition des œuvres. Cela va de l'acquisition des matériaux au transport des œuvres finies en passant par les transformations opérées sur ces matériaux et toutes les dépenses d'énergie fournies dans l'acte de création, sans oublier toutes les interactions qui résultent d'un travail collaboratif, souvent nécessaire. Tout cela n'est pas très éloigné de ce qu'on observe ailleurs, où les pratiques de création actuelles prennent le corps pour objet à un pourcentage avoisinant les $80 \%$, voire $90 \%$ (Michaud 2006; Ardenne 2010; Comar [dir.] 20 08). C e p oint de c onvergence u ne fo is relevé, il e st i mportant de constater que les voies par lesquelles les artistes y sont parvenus ne sont pas toujours les mêmes ici et ailleurs.

Prenons St.-Eloi. Il e st passé de la peinture de chevalet à l a fabrication d'objets volumétriques en utilisant des matériaux de récupération. II a trouvé et adopté cette solution au début des années 1990 en cherchant à contourner les difficultés à se procurer des tubes de peinture en raison de l'embargo é conomique imposé au pays suite au c oup d'État perpétré contre J ean-Bertrand A ristide ${ }^{10}$. À c ompter $d e c$ ette $d$ ate, il n e va $p$ lus s'approvisionner en tubes ou bocaux de peinture, en toiles apprêtées (ou à apprêter) et autres accessoires dans un magasin. II part à la recherche d'objets usagés dans le voisinage ou en d'autres endroits de la ville. Les lieux et les modes d'acquisition des matériaux changent, mais il faut également s'arranger pour leur stockage et se doter de tout le dispositif nécessaire à leur traitement. Les habitudes de travail ne sont plus les mêmes, depuis la conception des œuvres jusqu'aux procédures de réalisation, les postures et les gestes, la dépense physique, le temps de travail... II devient nécessaire d'en appeler à d'autres compétences. Benjamin, à l'occasion de la Fête de la sculpture de juillet 2007 à Port-au-Prince (Institut français d'Haïti-Fokal), 
dont il fut le commissaire, a formulé des observations sur St.-Eloi qui rassemblent matériaux utilisés, travail effectué, résultat obtenu et traces sur le corps. «Je suis impressionné, dit-il, par sa capacité d'insuffler une âme, une poésie à des matériaux extrêmement rudes. II travaille l'aluminium, il travaille le fer, il travaille l'acier, avec des effets de légèreté, d'élégance, de grâce, ce qui n'est pas du tout évident. J'ai vu ses mains pendant le festival, elles étaient extrêmement fatiguées. 》 (Lévy 2007: 133) Elles étaient encore bien abîmées lors de mes dernières rencontres avec l'artiste en novembre 2013.

Une d es i mplications $\mathrm{d} u \mathrm{p}$ assage $\mathrm{d} u \mathrm{t}$ ableau $\mathrm{d}$ e c hevalet a ux objets volumétriques est une forte tendance à l' individualisation ou l'autonomisation des figures, au profit de la représentation/présentation de corps. L'autonomisation v olumétrique d es p ersonnages su ppose, p our $\mathrm{S}$ t.-Eloi, de les détacher de l'espace pictural où ils é taient ha bituellement placés dans un environnement bien chargé (paysage, intérieur d'habitat, etc.) et pris dans des interactions, le plus souvent dans une composition à visée narrative. $L$ a figure a utonomisée a ppelle d es traitements de sur face o ù doivent être pris en compte les potentialités et les limites du matériau dans la recherche d'effets spécifiques. Tout cela aboutit à des résultats distincts de c eux obtenus par la peinture, même si le métal p rivilégié par St.-Eloi (l'aluminium) et son travail tout en finesse ne sont pas sans rapport avec la tonalité dominante de ses peintures (il a insisté, lors de l'un de nos entretiens, sur la difficulté qu'il avait surtout à se procurer du blanc) et le souci du détail qui s'y manifeste. Ses œuvres volumétriques sont à bien des égards comparables $\mathrm{p}$ ar I eur $\mathrm{t}$ raitement a ux o bjets d écoratifs o u d' orfèvrerie rencontrés dans ses peintures.

L'importance $p$ rise $p$ ar I a c réation v olumétrique e st I 'un d es f aits marquants de la scène qui s'affirme. Cela a sans doute joué dans l'intérêt porté au corps, ou dans la perception qu'on en tire, dans un univers où la peinture (le tableau) était et est encore (quantitativement) la pratique la plus développée. Mais cette importance est repérable aussi chez des artistes qui s'efforcent de faire advenir un type inédit de peinture. On conçoit que les démarches et les réalisations diffèrent. On observe toutefois l'apparition de thèmes, de sujets, de motifs, de procédés apparemment partagés par plusieurs artistes. Deux figures, celles du bizango et du gede, ont acquis une position qui semble nouvelle. La première est liée à une série d'objets de la collection de Marianne Lehmann ${ }^{\mathbf{1 1}}$, qui représenteraient des soldats de l'armée bizango (un type de société secrète). Ce seraient donc des chefs, des guerriers ou des esprits guerriers incarnés. Toute une série d'œuvres se sont développées en référence au monde des sociétés secrètes, avec couleurs sombres, cercueils, crânes, croix, fouets, armes blanches, clous, objets noués... Les gede, eux, sont les Iwa de la mort, du cimetière. Avec eux, c'est la perturbation, la débandade, le désordre, le sexe, l'obscénité, la grivoiserie, la dérision, mais également les liens indéfectibles entre la vie et la mort, le principe de la régénération. Ils gagnent les rues et vont dans les cimetières à la Toussaint, les $1^{\text {er }}$ et 2 novembre.

Si d es œ uvres t raitent explicitement d e c es figures, d 'autres qui , a priori, sont conçues dans de tout autres perspectives semblent partager avec elles un air de famille. Le rapprochement tient au traitement du corps
11. Cette collection a fait l'objet d'une exposition itinérante en Europe et en Amérique du Nord de 2007 à 2014 ; voir le catalogue édité par le musée d'Ethnographie de Genève (Hainard et Mathez [dir.] 2007). J'ai publié une analyse de la collection (Célius 2009d) dans laquelle je soutiens que mes recherches ne me permettent pas de confirmer l'ancienneté des statues bizango, comme on l'affirme. Mon hypothèse est que ces objets n'ont pas été conçus avant les années 1980-1990, ce qui me porte à les inscrire dans les mouvances de la nouvelle scène artistique, sans toutefois oublier les enjeux religieux et sociopolitiques internes dans lesquels la collection est prise. Sur la collection, voir BeauvoirDominique 2005 ; sur un bizango acheté par le musée du quai Branly, voir Delpuech 2011 et l'article de Benoît et Delpuech du présent volume, pp. 132-157. 


\section{ci-contre}

fig. 7

Pascale Monnin et

Lhérisson Dubréus, Notre Dame des Fleurs, 2011,

perles, paillettes, peinture sur toile. Avec l'aimable autorisation des artistes et de la Galerie Monnin. en proie à la violence, la difformité, la déchéance, la maladie, la mort, mais aussi à la mise en scène des sexes et des sexualités, ou encore de la dérision, du faux-semblant... Ainsi une tête énorme de Rocklor ou de Ti Pèlin aux traits prononcés, en pierre taillée, développe des affinités avec des peintures de Benjamin, plus précisément ce qu'il appelle ses «portraits psychologiques». Des installations du même Benjamin font écho aux bizango de la collection Lehmann (Pivin et al. 2012). De même, l'univers badin de Paskö, où se démultiplient les tete ("seins") et les kòk ("coq": le gallinacé, mais aussi I'organe sexuel masculin en c réole haïtien), et la série sur l'érection de Killy entrent en résonance avec une bonne partie de la production des artistes de I a G rand R ue p our l esquels I e p hallus e st r oi (fig. 5). C auvin montre que ce phallus roi implique aussi le viol et la prostitution dont sont victimes les femmes, notamment dans sa série sur les gangs. Certes, des contrepoints existent comme en atteste l'œuvre de Barthélemy qui, tout en suggérant, l ors de l'un de nos entretiens, que s es performances constituent $d$ es « mises à $n$ u ", n'est $p$ as $d$ ans u ne quelconque nudité littérale. Elle élabore une œuvre to ute en mesure, en évocation subtile. Y c ompris en abordant des drames (comme celui des corps de migrants échoués sur les plages de Miami) ou de hauts faits d'armes de héros nationaux (CapoisLa-Mort par exemple; fig. 4) où elle risque le pari de la disparition (de la non-représentation) des corps. Les aspects des traitements du corps qui viennent d'être évoqués interpellent, comme nous y i nvitent explicitement certaines œuvres, quant aux relations qui pourraient être établies avec des pratiques et des phénomènes qui affectent la société depuis la chute des Duvalier en 1986. Citons: les manifestations populaires récurrentes, les épidémies allant du sida au choléra, le développement de pratiques comme le kidnapping, la formation et l'affrontement de gangs, les fréquents assassinats, ciblés ou non, les viols, les trafics d'organes, les morts en nombre dues à des accidents et des catastrophes naturelles. Ajoutons la promiscuité des bidonvilles, l'explosion de la prostitution... Cette liste non exhaustive donne une idée d'un climat général de violence sur les corps dont on trouve des expressions, des descriptions, des échos dans des œuvres littéraires et musicales, offrant des analogies, à explorer, avec les œuvres plastiques.

\section{Techniques et matériaux}

La dynamique identifiée se distingue encore par bien d'autres aspects, dont le p lus significatif est sans d oute la recherche de voies inédites en dehors de la peinture, qui reste quantitativement la pratique artistique dominante dans le pays. Le volume et l'espace sont devenus plus préoccupants. Les jeux d'échelle explorés dans la conception des œuvres-objets invitent d'emblée à e nvisager leur mode d'inscription dans l'espace. C'est surtout dans les installations que les paramètres spatiaux sont conçus comme élément intégral de la création. Dans les performances aussi, où interviennent notamment la temporalité et les interactions directes avec le public. L'importance prise $p$ ar les o bjets volumétriques est $p$ articulièrement m arquante. Si on y retrouve des matériaux connus (bois, pierre, métal, béton...), l'exploration des potentialités de l'assemblage et de la récupération a bouleversé la donne. Le champ des possibles s'en trouve considérablement élargi, tant au n iveau d es m atériaux s usceptibles d'être u tilisés que d es te chniques et des savoir-faire. Le transfert de s avoir-faire, leurs confrontations, leurs juxtapositions, leurs mutualisations constituent l'un des soubassements de 


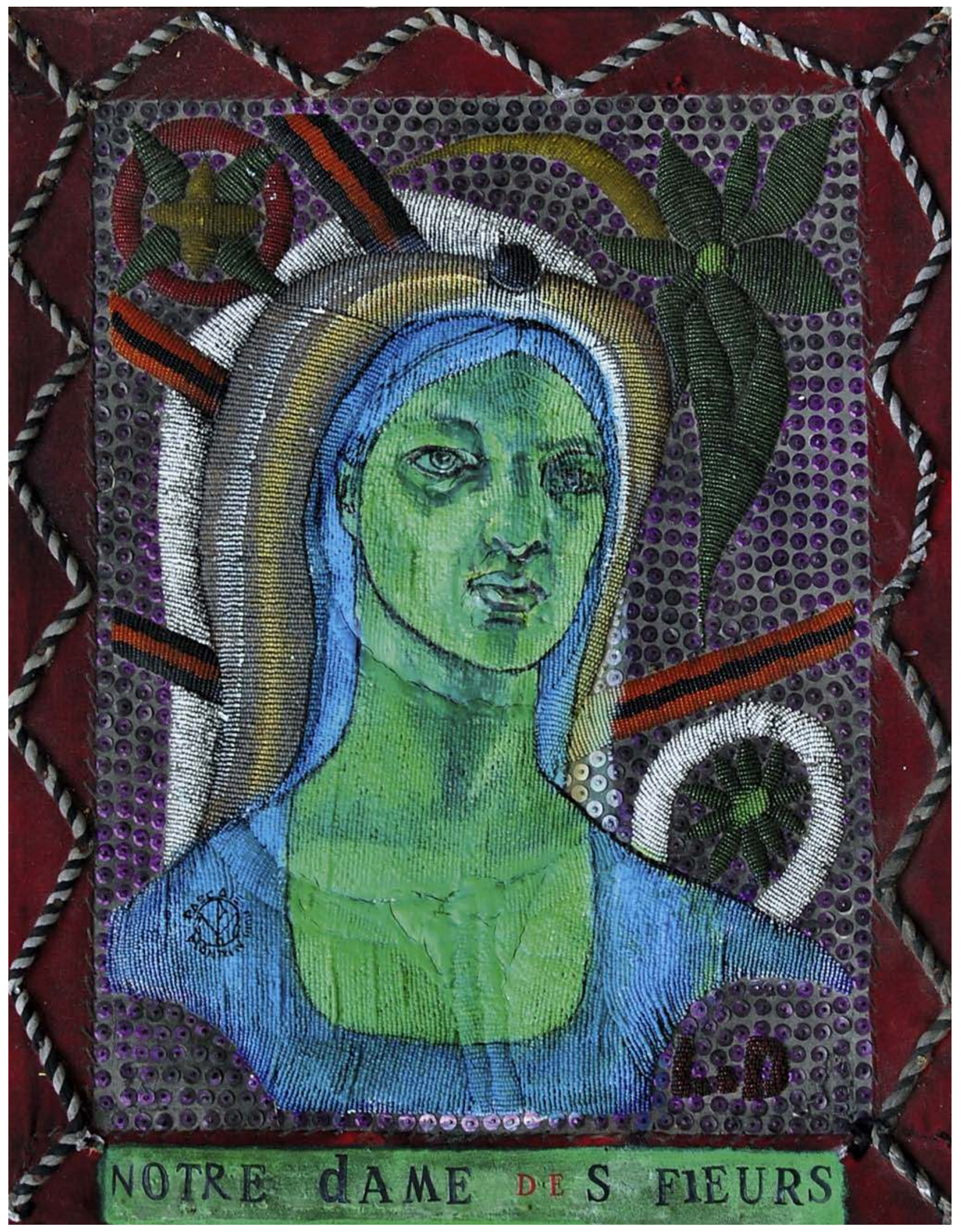




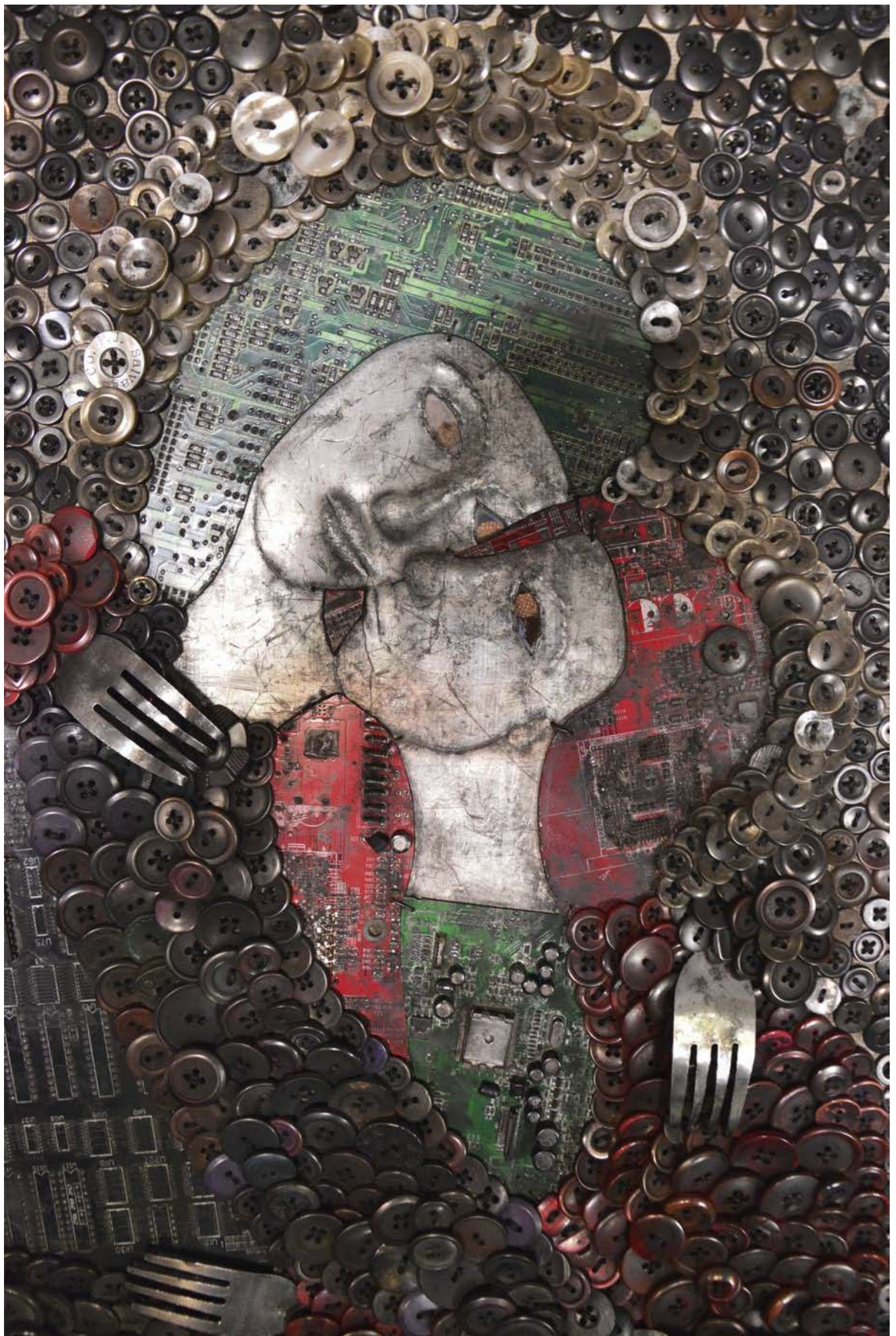


la nouvelle scène, l'un des ressorts de la créativité qui s'y manifeste. On le voit au niveau des démarches individuelles. Si des artistes peuvent déléguer la réalisation des œuvres en tout ou en partie, on assiste aussi à des échanges techniques formels entre la plupart d'entre eux, tout comme à des appropriations «clandestines». Des œuvres collaboratives ont été exécutées, comme Benjamin l'a expérimenté avec Nasson (qu'il a rencontré en $1987^{12}$ ) et avec les artistes de la Grand Rue (Guyodo, Céleur, Eugène) en 2006 (œuvre pour un char de carnaval et Freedom!, sculpture pour le Liverpool's International Slavery Museum). Monnin a signé une œuvre avec Lhérisson (fig. 7) et fait appel à une céramiste pour réaliser une partie d'une œuvre qui requiert sa compétence. St.-Eloi confie à un spécialiste de la soudure telle étape de son travail. Vilaire collabore avec des artisans qui fabriquent des ustensiles de cuisine à partir de la technique du coulage de l'aluminium. Au-delà du travail collaboratif, du partage et des transferts de savoir-faire, le phénomène décisif est la possibilité de soumettre matériaux et techniques à d es expérimentations, engendrant une démultiplication des opérations et des combinaisons. Les artistes peuvent nouer, filer, couler, clouer, clouter, ficeler, envelopper, coller, ajuster, casser, déchirer, prélever, mouler, trouer, tailler, souder, amarrer, recouvrir, flamber, gratter, décaper, découper, ciseler, agglomérer, juxtaposer, marteler, entoiler, repousser, empoussiérer, évider, coudre, découdre, colorer, enfumer, rembourrer...

Pour illustrer les processus de transformation à l'œuvre dans cette dynamique d' ensemble, a rrêtons-nous s ur l' exemple d es drapo v odou, bannières rituelles faites de perles et de paillettes ${ }^{\mathbf{1 3}}$, à partir du cas d'une artiste. Constant est parvenue à la fabrication d'objets de ce type par un biais d étourné. El le $n$ 'est $p$ as i ssue d' un d es a teliers qui e $n$ p roduisent. Sa technique est d'ailleurs tout à fait différente: elle ne coud pas les paillettes, les sequins et les perles sur du tissu, mais pratique le perlage. Elle fixe perles et paillettes, préalablement enfilées, au moyen d'un crochet (et non d'une aiguille, comme les autres), le revers face à elle (elle ne voit pas directement l'œuvre prendre forme, contrairement aux autres; voir l'article de Benson, fig. 6). II s'agit en fait de la technique de broderie perlée découlant de la broderie de Lunéville qu'elle a apprise de sa mère qui travaillait dans une usine de sous-traitance de fabrication d'objets perlés, des robes de mariage en particulier ${ }^{14}$. Après un passage à l'usine, elle emploie cette technique pour créer des œuvres s'apparentant aux drapo vodou.

Les objets rituels appelés drapo vodou étaient déjà entrés dans une dynamique de transformations induites par leur commercialisation. Une production non destinée aux cultes, nettement reconnaissable, s'est développée. Une iconographie inédite s'est déployée, débordant parfois les limites de la s ymbolique vodou. $\mathrm{D}$ ans $\mathrm{b}$ ien d es $\mathrm{c}$ as, I a peinture figurative e st $\mathrm{p}$ rise pour modèle, donnant lieu à toutes sortes de scènes narratives. Duval-Carrié a fait reproduire une série de ses peintures par des créateurs de ce type d'objet. C ompte tenu d e c es t ransformations, I'appellation drapo vodou, pour ce qui concerne cette veine créative, devient purement générique; elle renvoie aux usages initiaux des bannières et aux techniques utilisées plus qu'aux œuvres effectivement produites. J'avais déjà signalé, en esquissant les parcours de quelques artistes, que Barra, Lhérisson et Boyer viennent des a teliers de fabrication de ces objets. Barra a i ntroduit des pratiques
12. Selon la chronologie établie dans Pivin et al. 2012.

13. Sur les drapo vodou (histoire, fonction, analyse iconographique, description des techniques, profils de créateurs, implications de promoteurs, expositions...), on lira à profit Yonkers 1991 Girouard 1994, Newport 1997, Polk 1997 et Josephson 2007.

14. La broderie de Lunéville, apparue au XIX ${ }^{\ominus}$ siècle en France, consistait à imiter certaines dentelles par la broderie (sur tulle). Il existe encore, dans des pays disposant de mainsd'œuvre à bas coût, des ateliers de "petites mains " (brodeurs, brodeuses, perleuses...) qui, à l'aide de cette technique et d'autres, exécutent des motifs destinés aux maisons de haute couture. Voir Vulser 2012 pour des exemples en Inde.

\section{ci-contre}

fig. 8

Détail d'une œuvre de David Boyer, novembre 2013, dans l'atelier de l'artiste. (C) Photo Carlo A. Célius. 


\section{ci-contre}

fig. 9

Vladimir Cybil Charlier, Red Cross Tristan, 2009 paillettes et perles sur toile, impression jet d'encre et acrylique, bois. (C) Vladimir Cybil Charlier. d'assemblage où il transforme les drapo vodou en y ajoutant des éléments inhabituels comme des poupées importées (Cosentino 1998). II a aussi monté des pièces volumétriques recouvertes en tout ou en partie de paillettes. Sur s es traces, L hérisson et B oyer p oursuivent les recherches av ec u ne grande $\mathrm{c}$ réativité. B oyer a r emplacé I es $p$ aillettes $p$ ar d es b outons $q$ u'il confronte à d'autres o bjets, tels des circuits é lectroniques récupérés, et ajoute souvent des motifs (visages, formes humaines, a nimales) en métal repoussé (fig. 8). Cela ne s'arrête pas là. L'extension de l'usage des paillettes est manifeste dans des œuvres de Prézeau Stephenson, de Charlier (V for Victory, 2007 [fig. 9] ; Serenity and Punishment, 2003) ou encore de Guyodo (fig. 1). Ce dernier utilise des habits usagés importés (des kenedi ou encore des pèpè, comme on les désigne en Haïti) comportant ces éléments, qu'il découpe et applique sur ses objets volumétriques (fig. 2) ou sur des surfaces planes. Ce sont surtout les effets de scintillement qui importent ici, les mêmes qu'a cherché à rendre Killy, en pensant aux drapo vodou, par un procédé complètement différent. II applique sur du papier un composé pictural pouvant les restituer ou s'en approchant. Cette préoccupation rejoint la r echerche d'une s orte d' effet c inétique qu'explorent plusieurs a rtistes. Elle se manifeste par l'emploi de pièces de miroir, de billes, de verre, que l'on retrouve dans bien des œuvres. St.-Eloi découpe des tôles en aluminium pour obtenir de petites pièces de formes plus ou moins régulières, qui sont ensuite attachées les unes aux autres et recouvrent une structure, parfois de grande dimension (fig. 6). II e n ressort une surface comme en écailles de poisson qui rappelle l'assemblage des paillettes, qu'il a d'ailleurs pratiqué (Josephson 2007: 133-134). Sont ajoutés (parfois dans les petites pièces de tôle en aluminium ajouré) de nombreux morceaux de miroir, aux reflets démultipliés changeant selon l'angle et la distance de vue adoptés par le spectateur. Monnin, en plus des perles, des bijoux, parfois des miroirs, introduit la mobilité des œuvres suspendues avec des jeux de lumière et/ou un dispositif d'éclairage qui ajoute une «vitalité » supplémentaire par le jeu des ombres projetées des œuvres.

L'approche a doptée d ans c et a rticle et l es ex emples retenus p résentent l'intérêt de suggérer plusieurs pistes à explorer dans la perspective d'une étude approfondie de la nouvelle scène artistique d'Haïti. D'amples descriptions $v$ iendront $r$ estituer $I$ es $d$ ifférents a spects $d$ es $d$ ynamiques à l'œuvre e n c herchant, c omme j' ai te nté d e le faire i ci, à to ujours te nir ensemble, à a rticuler p lusieurs é chelles d' analyse. Pa rmi I es p oints q ui semblent dé terminants pour la suite, soulignons la prise en compte des relations internes (le pays)/externes (l'étranger), le s m odes de s tructuration et de restructuration internes de l'univers de la création plastique, les stratégies des différents acteurs impliqués, dont les créateurs eux-mêmes, et évidemment les caractéristiques des œuvres qui résultent de tout cela... De c e p oint d e v ue, l' exemple d es drapo v odou, p our I imité q u'il s oit, revêt un caractère emblématique. Il exemplifie la visibilité et la valorisation d'une pratique relevant d'un domaine spécifique de création, l'impact du marché et les transformations qui en résultent, les transferts de savoir-faire, de potentialités créatrices découvertes et explorées. 


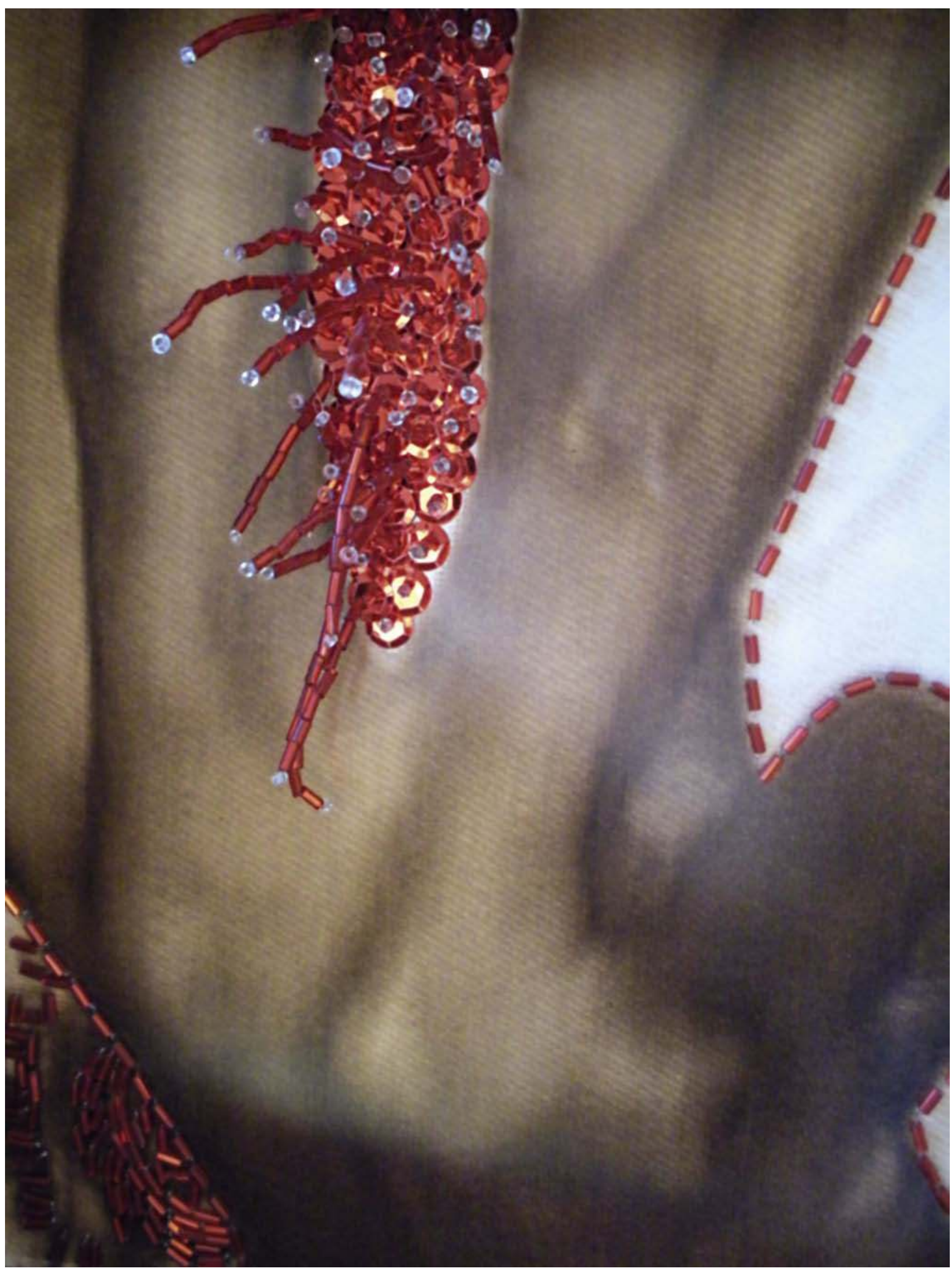


Bibliographie

\section{Alexis, Gérald}

2000 Peintres haitiens.

Paris, Éditions Cercle d'Art.

\section{Ardenne, Paul}

2010 L'Image corps. Figures de l'humain dans l'art du xx' siècle. Paris, Éditions du Regard.

\section{Bambou, Pierre}

1974 "Le concept d'oraliture", Le Nouvelliste, 12 mai.

\section{Beauvoir-Dominique, Rachel}

2005 "Libérer le double, la beauté sera convulsive... À propos d'une collection d'art vaudou ", Gradhiva (nouvelle série) 1: 57-69.

\section{Belting, Hans}

1998 [1990] /mage et culte.

Une histoire de l'image avant l'époque de l'art, trad. de l'allemand par Frank Muller. Paris, Éditions du Cerf.

\section{Cadet, Jean-Francois}

2013 Entretien avec Mario Benjamin, émission Vous m'en direz des nouvelles!, RFI, 11 janvier.

\section{Célius, Carlo A.}

2000 «Les enjeux de la représentation. Portraits de Noirs et de mulatres pendant la Révolution à Saint-Domingue (1789-1804) ", in Berta Ares Queija et Alessandro Stella (dir.), Negros, Mulatos, Zambaigos. Derroteros africanos en los mundos ibéricos. Séville, Escuela de Estudios HispanoAmericanos-Consejo Superior de Investigaciones Científicas: 313-360.

2004 «L'avènement de l'art naiif en Haïti. La portée instauratrice d'un jugement esthétique", RACAR [Revue d'art canadienne/Canadian Art Review], XIX (1-2): 47-64 [parution juillet 2005].

2006 «Saint Fleurant dans la mêlée», in Louisiane Saint Fleurant, catalogue d'exposition aux Ateliers Jérôme et à l'Institut français d'Haïti. Port-auPrince, Les Éditions Areytos: 9-13.

2007a Langage plastique et énonciation identitaire. L'invention de l'art haitien. Québec, Les Presses de l'université Laval. 2007b «Sous le signe d'Ayizan. Introduction à l'univers iconique de Tiga ", in Tiga, Moulé dans l'argile, catalogue d'exposition. Port-auPrince: 43-57 [éditeur non indiqué dans l'ouvrage].

2008 «Considérations sur linvention des vèvè ", in Jacques Hainard, Philippe Mathez et Olivier Schinz (dir.), Vodou. Gollion et Genève, Infolio éditions-Musée d'Ethnographie de Genève («Tabou 5 »): 279-305.

2009a «Neoclassicism and the Haitian Revolution ", in David Patrick Geggus et Norman Fiering (éd.), The World of Haitian Revolution. Bloomington et Indianapolis, Indiana University Press: 352-392.

2009b " "Lart" en situation coloniale esclavagiste. Saint-Domingue, xvile siècle ", Cahiers des Anneaux de la mémoire 12 : 61-83.

$2009 c$ «Célestin Faustin, un peintre haïtien face au sacré ", Histoire et missions chrétiennes 12: 93-110.

2009d "Le vodou haïtien: patrimonialisation et dynamique de restructuration », Martor, revue d'anthropologie du musée du paysan roumain 14: 89-100.

2011 "Les vèvè du créateur ", in Hector Hyppolite. Paris, Éditions de Capri: 67-98.

2014 «L'imagerie chrétienne dans la création plastique d'Haïti», Histoire, monde et cultures religieuses $29: 143-172$.

\section{Célius, Carlo A. (dir.)}

2005 Haiti et l'anthropologie, Gradhiva (nouvelle série) 1.

\section{Charlier, Vladimir Gybil}

2005 «When the Saints go marching by ", Small Axe IX (2) : 52-55.

\section{Clorméus, Léwis Ampidu}

2012 Entre l'État, les élites et les religions en Haitti. Redécouvrir la campagne antisuperstitieuse de 1939-1942, thèse de doctorat en sociologie, École des hautes études en sciences sociales-université d'État d'Haîti.

\section{Comar, Philippe (dir.)}

2008 Figures du corps. Une leçon d'anatomie à l'école des Beaux-Arts. Paris, Éditions de l'ENSBA.

\section{Cometti, Jean-Pierre}

2012 Art et facteurs d'art. Ontologies friables. Rennes, Presses universitaires de Rennes.

\section{Cosentino, Donald J.}

1998 Vodou Things. The Art of Pierrot Barra and Marie Cassaise. Jackson, University Press of Mississippi.

Cosentino, Donald J. (éd.)

1998 [1995] Sacred Arts of Haitian Vodou. Los Angeles, UCLA Fowler Museum of Cultural History.

\section{Delpuech, André}

2011 «Un personnage bizango d'Haïti », in J.M.G. Le Clézio, Les musées sont des mondes. Paris, Gallimard-Musée du Louvre Éditions : 86-90.

\section{Dumas, Pierre-Raymond}

1984 «Interview sur le concept d'oraliture accordée à PierreRaymond Dumas par le Dr Ernest Mirville ", Conjonction 161-162 : 161-164.

\section{Girouard, Tina}

1994 Sequin Artists of Haiti. La Nouvelle-Orléans, Contemporary Arts Center.

\section{Hainard, Jacques et Mathez, Philippe (dir.)}

2007 Le Vodou, un art de vivre. Gollion et Genève, Infolio-Musée d'Ethnographie de Genève.

\section{Hall, Stuart}

2007 Identités et cultures. Politiques des cultural studies. Paris, Éditions Amsterdam.

2013 Identités et cultures 2. Politiques des différences. Paris, Éditions Amsterdam.

\section{Josephson, Nancy}

2007 Spirits in Sequins. Vodou Flags of Haiti. Atglen, Schiffer Publishing Ltd.

\section{Laroche, Maximilien}

1991 La Double Scène de la représentation. Oraliture et littérature dans la Caraïbe. Québec, GRELCAUniversité Laval.

\section{Lerebours, Michel-Philippe}

1989 Haïti et ses peintres de 1804 à 1980. Souffrances et espoirs d'un people. Port-au-Prince, Imprimeur II, 2 volumes.

\section{Lévy, Paul Élie}

$\mathbf{2 0 0 7}$ «ntretien avec Mario Benjamin ", in Fête de la sculpture. Port-au-Prince, Institut francais d'Haïti-Fokal : 128-139.

\section{Michaud, Yves}

2006 «Visualisations. Le corps et les arts visuels", in Alain Corbin, Jean-Jacques Courtine et Georges Vigarello (dir.), Histoire du corps, t. III, Les mutations du regard. Paris, Seuil : 417-436.

\section{Moreau de Saint-Méry, Louis-Élie}

1784-1790 Loix et constitutions des colonies françoises de l'Amérique sous le Vent. Tome quatrième comprenant les loix et constitutions depuis 1750 jusqu'en 1765 inclusivement. À Paris, l'auteur.

\section{Newport, Mark}

1997 Heralding the Divine Horsemen. Haitian Vodou Banners. Toronto, Museum for Textiles.

\section{Pivin, Jean Loup et al.}

2012 La Chambre de Mario Benjamin/The Room of Mario Benjamin. Paris, Revue Noire Éditions.

\section{Polk, Patrick Arthur}

1997 Haitian Vodou Flags. Jackson, University Press of Mississippi. 


\section{Ramsey, Kate}

2011 The Spirits and the Law. Vodou and Power in Haiti. Chicago et Londres, The Chicago University Press.

\section{Schaeffer, Jean-Marie}

2008 "Le musée du quai Branly entre art et esthétique ", Le Débat 148: 170-178.

2009 Théorie des signaux coûteux, esthétique et art. Rimouski,

Tangence Éditeur.

\section{Vulser, Nicole}

2012 "Les Indiens, petites mains du luxe ", Le Monde, 7 septembre.

\section{Yonkers, Dolores}

1991 Sequined Surfaces: Vodun Flags from Haïti. Northridge, Art Galleries of California State University. page 104 et ci-contre Maksaens Denis, My Joshua Tree, 2008. Installation vidéo. ( ) Maksaens Denis.

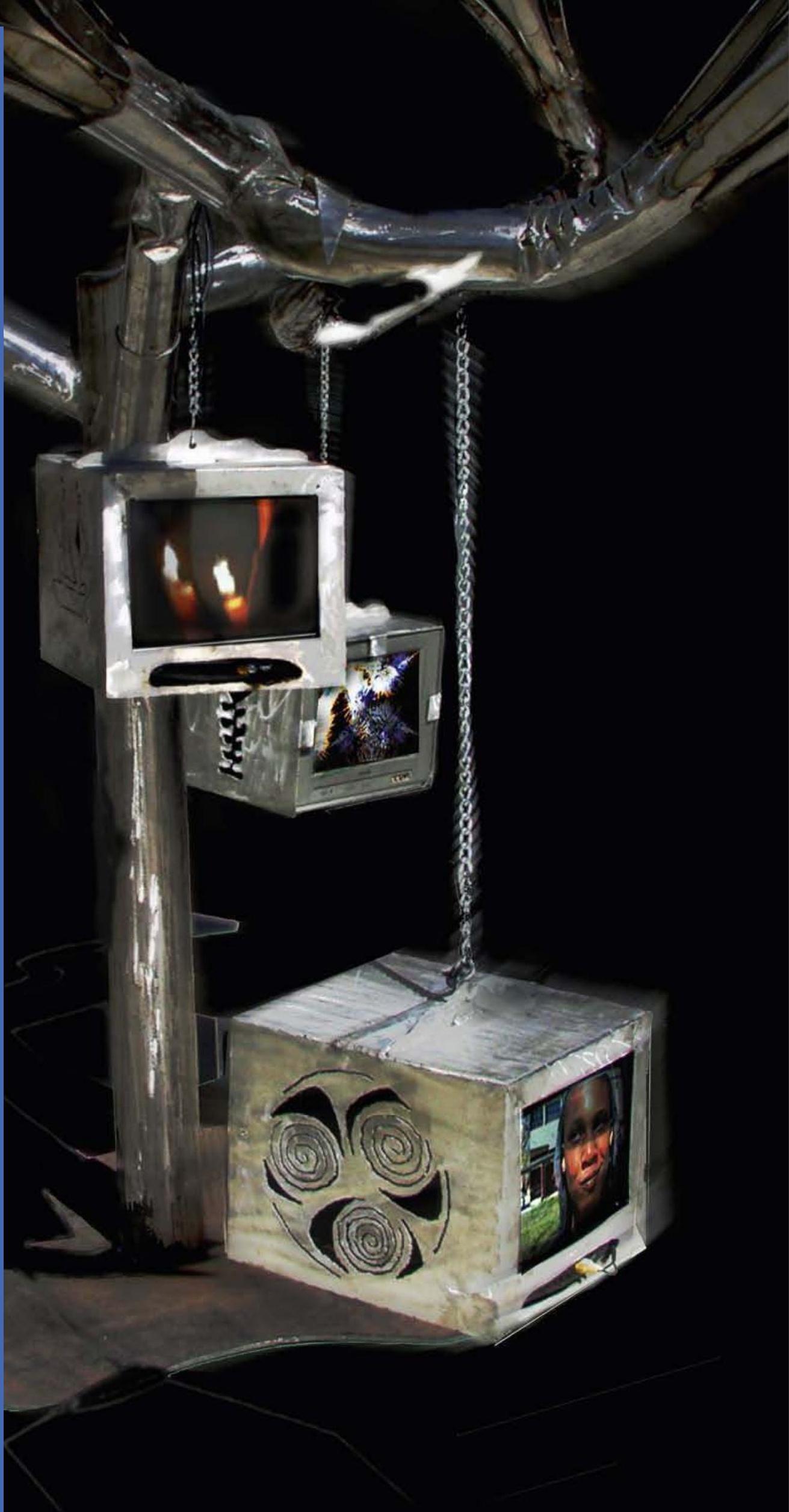

